\title{
A new approach in the determination of characteristic directions of the geoelectric structure using Mohr circles
}

\author{
John Makris, Nikos Bogris, and Kostas Eftaxias \\ Solid Earth Physics Institute, University of Athens, Panepistimiopolis, Zografos, 15784, Greece
}

(Received November 2, 1998; Revised February 23, 1999; Accepted April 12, 1999)

\begin{abstract}
The use of Mohr circles into magnetotelluric (MT) intepretation was introduced by Lilley (Lilley, 1976). By plotting $Z_{x x}$-rotated versus $Z_{x y}$-rotated (real and imaginary parts) important information on the conductivity structure is obtained. In this paper Mohr circles are employed to reveal the directions of polarisation of the electric field. Furthermore, the following procedure for Mohr circle analysis is suggested: plotting $Z_{x x}$-rotated versus $Z_{y x}$-rotated (instead of $Z_{x y}$-rotated) the principal axes system i.e., the strike angle, of the regional 2D structure can be resolved in some cases. The latter analysis is implemented to MT data from Ioannina area, in NW Greece. Results from other intepretation techniques applied to the same dataset, such as decomposition methods, tipper and induction arrows analysis, provide support for the validity of the conclusions reached.
\end{abstract}

\section{Introduction}

During the last two decades an extensive experimental study in Greece (Varotsos et al., 1996a) showed that earthquakes are preceded by transient electric signals called preSeismic Electric Signals (SES). One of the main results of this research indicates that SES are detected only at certain sites (sensitive points) of the Earth's surface. Recent theoretical studies (Varotsos et al., 1998) suggest that the SES sensitivity of a site should be connected to the geoelectric structure. In an effort to investigate whether such a connection actually exists, we performed a detailed magnetotelluric study of an area near to Ioannina city which, after a long experimentation, has been found to be SES sensitive. It is far from the scope of this paper to review the results of this detailed MT analysis but only to report on a new approach for the determination of characteristic directions of the geoelectric structure using Mohr circles. The paper is organised as follows: Section 2 reviews the magnetotelluric representation when Mohr circles are employed. Section 3 we first present such an analysis of the MT data of Ioannina area, and then proceed to the suggestion of a new form of the magnetotelluric representation of Mohr circles. Finally, in Section 4 conclusions are drawn by comparing the results obtained from different ways of analysis.

\section{Magnetotellurics and Mohr Circles}

Mohr circles are widely used for analysing stress variation and finite strain in bodies (Nye, 1957). The technique was introduced to magnetotellurics by Lilley (Lilley, 1976). In magnetotellurics, Mohr circles illustrate the variation of real or imaginary part of element $Z_{x x}$ of the impedance tensor versus the real or imaginary part of element $Z_{x y}$, as the hor-

Copy right (C) The Society of Geomagnetism and Earth, Planetary and Space Sciences (SGEPSS); The Seismological Society of Japan; The Volcanological Society of Japan; The Geodetic Society of Japan; The Japanese Society for Planetary Sciences. izontal axes of the measuring coordinate system are rotated clockwise through an angle $\theta,\left(0^{\circ} \leq \theta \leq 180^{\circ}\right)$. This presentation enables the display of important information from the impedance tensor concerning the dimensionality of the geoelectric structure and the decomposition model parameters.

Let us consider the measured impedance tensor, $\mathbf{Z}_{\mathbf{m}}$. A clockwise rotation of the measuring coordinate system $(x, y)$ through an angle $\theta$, produces a new measuring coordinate system $\left(x^{\prime}, y^{\prime}\right)$. At this system, the elements $Z_{i j}^{\prime}$ of the rotated impedance tensor $\mathbf{Z}_{\mathbf{m}}^{\prime}(\theta)$ are determined from the expansion of the equation:

$$
\mathbf{Z}^{\prime}(\theta)=\mathbf{R}(\theta) \mathbf{Z}_{\mathbf{m}} \mathbf{R}^{\mathbf{T}}(\theta)
$$

where $\mathbf{R}(\theta)$, is the rotational operator for an angle $\theta$.

The analytical expressions obtained by virtue of (1) are the following:

$$
\begin{aligned}
Z_{x x}^{\prime}= & \frac{\left(Z_{x x}+Z_{y y}\right)}{2}+\frac{\left(Z_{x y}+Z_{y x}\right)}{2} \sin 2 \theta \\
& +\frac{\left(Z_{x x}-Z_{y y}\right)}{2} \cos 2 \theta, \\
Z_{x y}^{\prime}= & \frac{\left(Z_{x y}-Z_{y x}\right)}{2}+\frac{\left(Z_{x y}+Z_{y x}\right)}{2} \cos 2 \theta \\
& -\frac{\left(Z_{x x}-Z_{y y}\right)}{2} \sin 2 \theta, \\
Z_{y x}^{\prime}= & -\frac{\left(Z_{x y}-Z_{y x}\right)}{2}+\frac{\left(Z_{x y}+Z_{y x}\right)}{2} \cos 2 \theta \\
& -\frac{\left(Z_{x x}-Z_{y y}\right)}{2} \sin 2 \theta .
\end{aligned}
$$

By taking the real parts of the complex quantities $Z_{i j}$, the combination of Eqs. (2) and (3) leads to:

$$
\begin{aligned}
& \left(Z_{x x_{r}}^{\prime}(\theta)-Z_{2_{r}}\right)^{2}+\left(Z_{x y_{r}}^{\prime}(\theta)-Z_{1_{r}}\right)^{2} \\
& \quad=\frac{\left(Z_{x y_{r}}+Z_{y x_{r}}\right)^{2}}{4}+\frac{\left(Z_{x x_{r}}-Z_{y y_{r}}\right)^{2}}{4}
\end{aligned}
$$




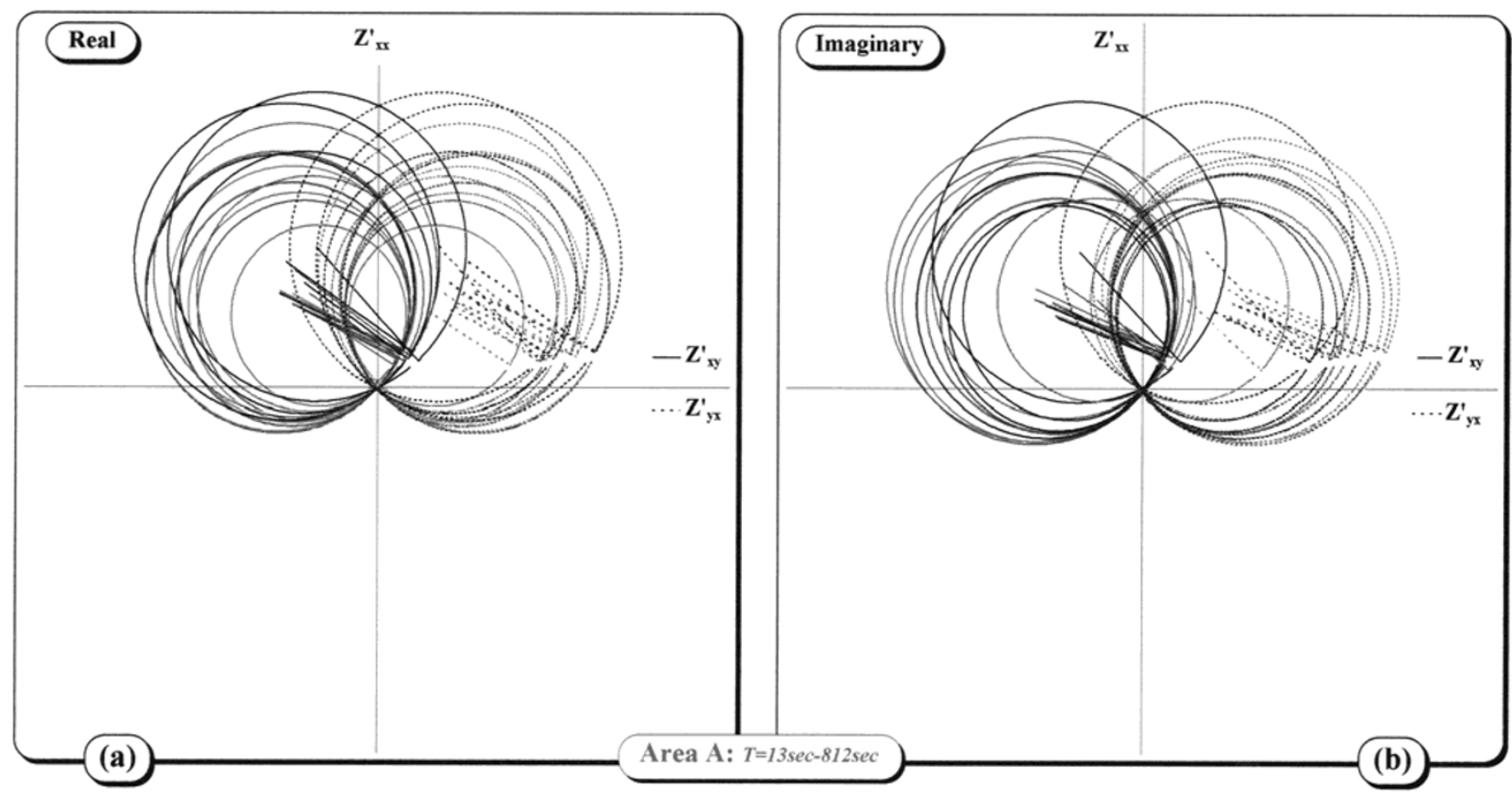

Fig. 1. Mohr circles at various periods, by taking (a) the real and (b) the imaginary parts of the $Z_{x x}^{\prime}$ vs $Z_{x y}^{\prime}$ impedance tensor elements (circles to the left of the diagrams (a) and (b)) and of the $Z_{x x}^{\prime}$ vs $Z_{y x}^{\prime}$ impedance tensor elements (circles to the right of the diagrams (a) and (b)), determined from MT data from the site A of Ioannina region (cf. for each circle, a line connects the center with the respective first point, which corresponds to a rotation angle equal to $0^{\circ}$. Note that the two points which correspond to the rotation angles $179^{\circ}$ and $180^{\circ}$ respectively, are intentionally omitted in order to depict the counterclockwise way of circle construction).

where the rotationally invariant quantities $Z_{1_{r}}$ and $Z_{2_{r}}$ are defined by:

$$
\begin{aligned}
& Z_{1_{r}}=\frac{\left(Z_{x y_{r}}-Z_{y x_{r}}\right)}{2}, \\
& Z_{2_{r}}=\frac{\left(Z_{x x_{r}}+Z_{y y_{r}}\right)}{2},
\end{aligned}
$$

respectively. Equation (5) indicates that a circle results when plotting $Z_{x x}^{\prime}(\theta)$ versus $Z_{x y}^{\prime}(\theta)$ upon varying the rotational angle $\theta$. This circle is characterised by the following parameters:

Center coordinates: $Z_{1 r}, Z_{2 r}$,

Radius: $R=\frac{1}{2}\left[\left(Z_{x y_{r}}+Z_{y x_{r}}\right)^{2}+\left(Z_{x x_{r}}-Z_{y y_{r}}\right)^{2}\right]^{1 / 2}$.

If a point $\mathrm{A}\left(Z_{x y_{r}}, Z_{x x_{r}}\right)$ of the circle refers to the measuring system, then a clockwise rotation of the measuring system through an angle $\theta$ reflects to a counterclockwise angular displacement $2 \theta$ of the point $\mathrm{A}$ on the circumference of the circle. Thus the new coordinates of A are given by $\left(Z_{x y_{r}}^{\prime}, Z_{x x_{r}}^{\prime}\right)$.

In the case of 1D-symmetry of the geoelectric structure the Mohr circle degenerates to a point which lies on the $Z_{x y_{r}}^{\prime}$ axis, while in the case of 2D-symmetry the Mohr circle center also lies on the $Z_{x y_{r}}^{\prime}$ axis but has a certain radius. In the case of 3D-symmetry the center of Mohr circle does not lie on the horizontal axis. When near surface local inhomogeneities are present over an 1D or 2D regional structure (Lilley, 1993) the symmetry of the impedance tensor is lost and the circle exhibits an angular offset that may be regarded as a skew angle.

In this paper we suggest the study of a "conjugate" form of these circles by substituting the element $Z_{x y}^{\prime}(\theta)$ with the element $Z_{y x}^{\prime}(\theta)$ to the construction of the relevant Mohr circle (Makris, 1997; Makris et al., 1997). Such a representation, as will be shown below, allows, in some cases, the determination of an additional important information of the geoelectric structure (i.e., the regional strike angle).

\section{Data Collection and Analysis}

The analysis of Mohr circles is applied to the data of an area which, after a long experimentation, was found to be sensitive for the detection of Seismic Electric Signals (SES) that are detected prior to earthquakes. This area lies in NW Greece near to Ioannina city. MT data from three sites called A, B and C (Varotsos et al., 1996a,b) were used. Figures $1-3$ depict the Mohr circles, $Z_{x x}^{\prime}(\theta)$ vs $Z_{x y}^{\prime}(\theta)$, (solid line circle groups) constructed separately for the real and imaginary parts. An inspection of these figures shows that, for the frequency band under consideration (i.e., 10-1000 s), all the circles pass approximately through the origin $\left(Z_{x y_{k}}^{\prime}, Z_{x x_{k}}^{\prime}\right)$, where $k=r, i$ denote the real and imaginary parts respectively. This peculiarity indicates that if we rotate clockwise the measuring system (i.e., $\mathrm{x}$-axis $\rightarrow \mathrm{NS}$, y-axis $\rightarrow \mathrm{EW}$ ) by an angle $\theta_{l}$, i.e., $\left(x_{\theta_{l}}^{\prime}, y_{\theta_{l}}^{\prime}\right)$, the elements of the first row of the (rotated) impedance tensor $\mathbf{Z}^{\prime}\left(\theta_{\mathbf{l}}\right)=\mathbf{R}\left(\theta_{\mathbf{l}}\right) \mathbf{Z}_{\mathbf{m}} \mathbf{R}^{\mathbf{T}}\left(\theta_{\mathbf{l}}\right)$ become both approximately zero:

$$
E_{x_{\theta_{l}}^{\prime}}=Z_{x x}^{\prime}\left(\theta_{l}\right) H_{x_{\theta_{l}}^{\prime}}+Z_{x y}^{\prime}\left(\theta_{l}\right) H_{y_{\theta_{l}}^{\prime}}=0
$$

which physically means that irrespective of the polarisation of the incident magnetic field, the electric field is linearly polarised in the direction of $y_{\theta_{l}}^{\prime}$-axis. These rotation angles were also calculated analytically from the representations 


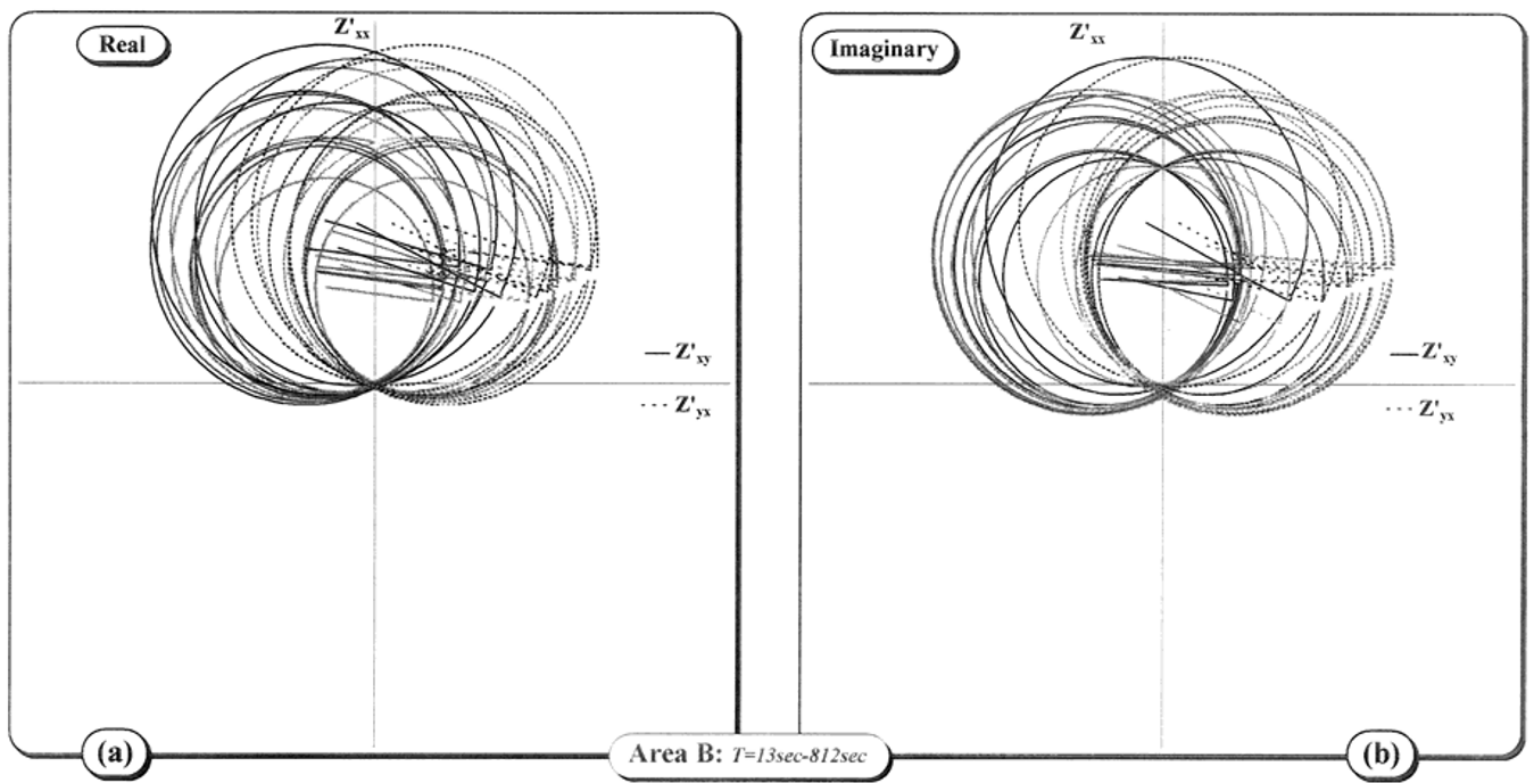

Fig. 2. Mohr circles at various periods, by taking (a) the real and (b) the imaginary parts of the $Z_{x x}^{\prime}$ vs $Z_{x y}^{\prime}$ impedace tensor elements (circles to the left of the diagrams (a) and (b)) and of the $Z_{x x}^{\prime}$ vs $Z_{y x}^{\prime}$ impedance tensor elements (circles to the right of the diagrams (a) and (b)) determined from MT data from the site $B$ of Ioannina region (see also the clarification of Fig. 1).

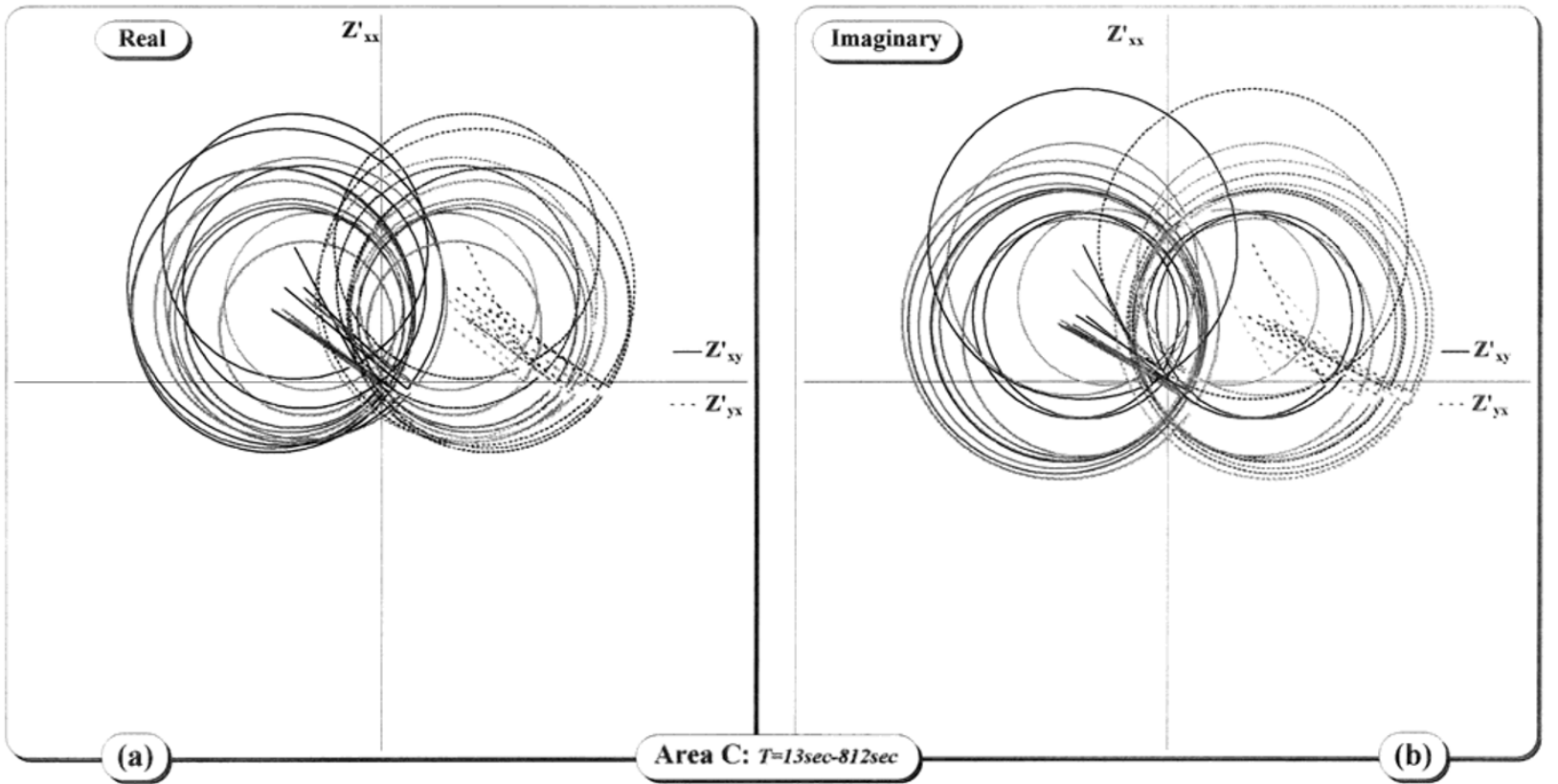

Fig. 3. Mohr circles at various periods, by taking (a) the real and (b) the imaginary parts of the $Z_{x x}^{\prime}$ vs $Z_{x y}^{\prime}$ impedace tensor elements (circles to the left of the diagrams (a) and (b)) and of the $Z_{x x}^{\prime}$ vs $Z_{y x}^{\prime}$ impedance tensor elements (circles to the right of the diagrams (a) and (b)) determined from MT data from the site $\mathrm{C}$ of Ioannina region (see also the clarification of Fig. 1).

(for the real and imaginary parts) of the Mohr circles for each of the sites A, B and C by minimising the quantity:

$$
\left.\sqrt{\left[Z_{x x}^{\prime}(\theta)\right]^{2}+\left[Z_{x y}^{\prime}(\theta)\right]^{2}}\right|_{\theta=\theta_{l}}
$$

and are plotted versus the period in Fig. 4. This figure indicates that the local electric field must be strongly linearly polarised in the directions $\sim \mathrm{N} 80^{\circ} \mathrm{E}, \sim \mathrm{N} 60^{\circ} \mathrm{E}, \sim \mathrm{N} 90^{\circ} \mathrm{E}$ at the sites $\mathrm{A}, \mathrm{B}$ and $\mathrm{C}$ respectively; this results from the fact that these angles (taking either the real or the imaginary parts) were found to be $\theta_{l}^{A} \simeq 170^{\circ}, \theta_{l}^{B} \simeq 150^{\circ}$ and $\theta_{l}^{C} \simeq 180^{\circ}$ for the sites $\mathrm{A}, \mathrm{B}$ and $\mathrm{C}$ respectively. An independent verification comes from the electric field polarisation diagrams (Fig. 5) which are constructed by using the raw data; they show that the electric field, at all the sites A, B and C, exhibits strong linear polarisation but in different directions, i.e., $\sim \mathrm{N} 86^{\circ} \mathrm{E}, \sim \mathrm{N} 62^{\circ} \mathrm{E}$ and $\sim \mathrm{N} 87^{\circ} \mathrm{E}$ respectively, which 

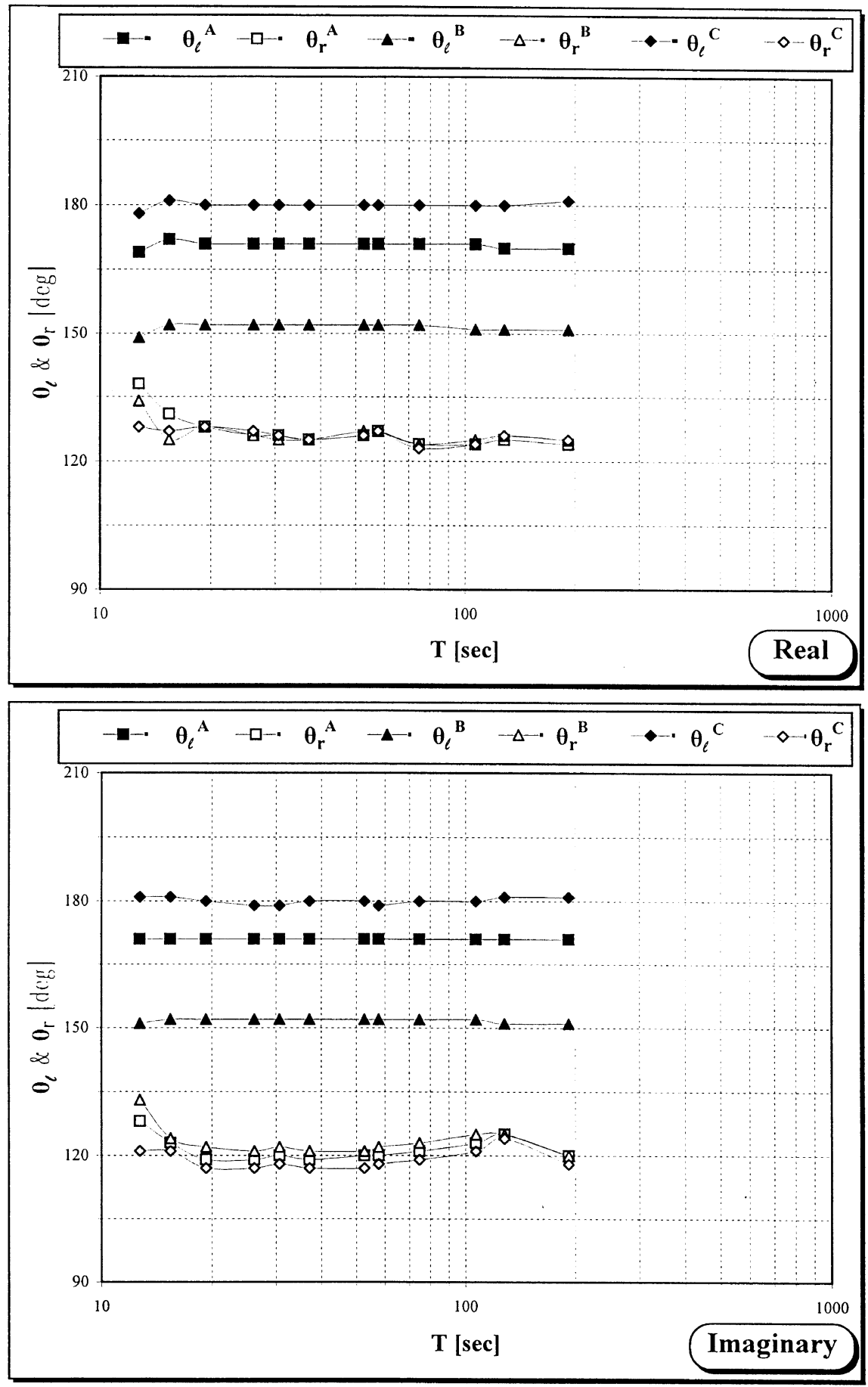

Fig. 4. The polarisation angle of the electric field $\theta_{l}$ and the regional strike angle $\theta_{r}$ versus the period for the sites A, B and C of the Ioannina region obtained from the Mohr circles that have been constructed using either the real or the imaginary parts of $Z_{x x}^{\prime}$ vs $Z_{x y}^{\prime}$ and $Z_{x x}^{\prime}$ vs $Z_{y x}^{\prime}$ respectively.

agrees with the values mentioned above. Note that approximately, the same angles have been independently determined (as local channelling directions, i.e., $\sim \mathrm{N} 81^{\circ} \mathrm{E}, \sim \mathrm{N} 62^{\circ} \mathrm{E}$ and $\sim \mathrm{N} 90^{\circ} \mathrm{E}$ respectively), when Groom-Bailey decomposition methodology (Groom and Bailey, 1989) is implemented on the experimental data (Makris, 1997; Makris et al., 1997). Moreover, further results from our analysis suggest the existence of local channelling for the geoelectric structure, as it will be discussed below.

We now turn to the "conjugate" form of the magnetotel- luric representation of Mohr circles, which has been used by Varotsos et al. (1999) to disclose more clearly the geoelectric structure at Ioannina along with the determination of the extent of the SES sensitive site. The axes of the measuring coordinate system $(\mathrm{x} \rightarrow \mathrm{NS}, \mathrm{y} \rightarrow \mathrm{EW})$ are rotated clockwise by the angle $\theta$ (where $0^{\circ} \leq \theta \leq 180^{\circ}$ ) and the variation of the real (respectively imaginary) part of the impedance tensor element $Z_{x x}^{\prime}(\theta)$ is plotted versus the element $Z_{y x}^{\prime}(\theta)$ (instead of representing the variation of the real (imaginary) part of element $Z_{x x}^{\prime}(\theta)$ versus the element $Z_{x y}^{\prime}(\theta)$ ). Fig- 

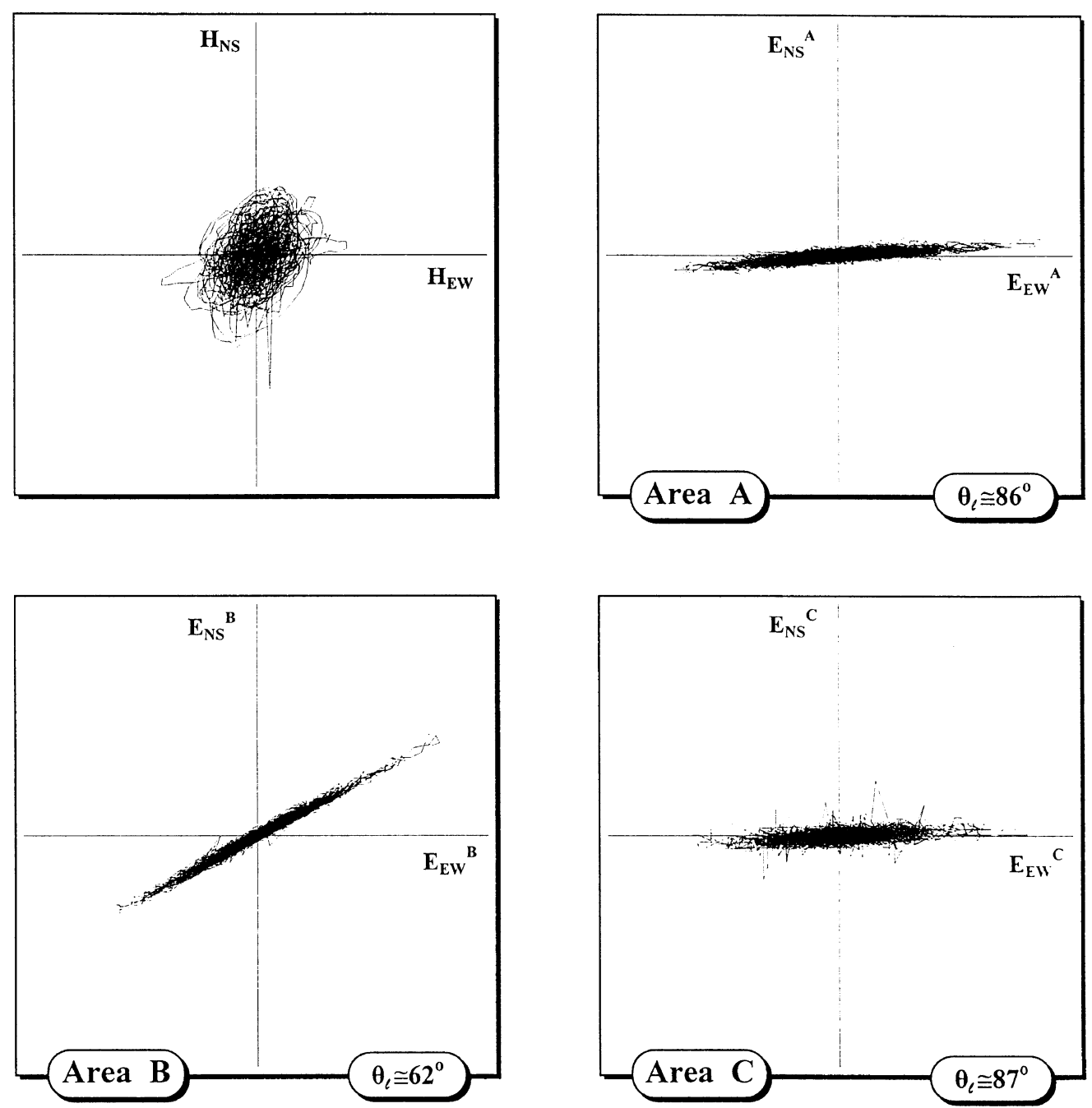

Fig. 5. Experimental polarisation diagrams of the electric field measured at the sites $\mathrm{A}, \mathrm{B}$ and $\mathrm{C}$ of Ioannina region. The local polarisation angle (clockwise from NS direction) of the electric field is depicted. For the sake of comparison, the same diagram, for the magnetic field is also given.

ures 1-3 depict the relevant Mohr circles (dashed-line circle groups), taking the real and the imaginary parts separately, for the same periods and for each one of the sites A, B and C. These circles exhibit the same peculiarity, i.e., they all pass through the origin of the axes $\left(Z_{y x}^{\prime}, Z_{x x}^{\prime}\right)$, irrespective of the measuring site, the period and the consideration of either the real or imaginary parts. This behaviour is expected, since taking Eqs. (2) and (4) instead of (2) and (3) it is clear that the "conjugate" Mohr circle is just exactly a mirror image of the standard circle with respect to the vertical coordinate axis. This characteristic implies that, if we rotate clockwise the measuring system $\left(x_{\theta_{r}}^{\prime}, y_{\theta_{r}}^{\prime}\right)$ by an angle $\theta_{r}$, the elements of the first column of the (rotated) impedance tensor $\mathbf{Z}^{\prime}\left(\theta_{\mathbf{r}}\right)=\mathbf{R}\left(\theta_{\mathbf{r}}\right) \mathbf{Z}_{\mathbf{m}} \mathbf{R}^{\mathbf{T}}\left(\theta_{\mathbf{r}}\right)$, i.e., $Z_{x x}^{\prime}\left(\theta_{r}\right)$ and $Z_{y x}^{\prime}\left(\theta_{r}\right)$, both become approximately zero. By recalling that:

$$
\begin{aligned}
& E_{x_{\theta_{r}}^{\prime}}=Z_{x x}^{\prime}\left(\theta_{r}\right) H_{x_{\theta_{r}}^{\prime}}+Z_{x y}^{\prime}\left(\theta_{r}\right) H_{y_{\theta_{r}}^{\prime}}, \\
& E_{y_{\theta_{r}}^{\prime}}=Z_{y x}^{\prime}\left(\theta_{r}\right) H_{x_{\theta_{r}}^{\prime}}+Z_{y y}^{\prime}\left(\theta_{r}\right) H_{y_{\theta_{r}}^{\prime}},
\end{aligned}
$$

we may reach the following physical explanation: the incidence of a magnetic field linearly polarised in the direction $x_{\theta_{r}}^{\prime}$ does not practically induce electric field; this result refers to the $H$-polarisation mode of a highly anisotropical 2 D regional structure (e.g., a vertical conductivity boundary), where the measuring site lies on the conductive medium but close to the resistivity contrast (d'Erceville and Kunetz, 1962; Swift, 1971; Fischer et al., 1992). In this case the magnetic field polarisation direction indicates the $2 \mathrm{D}$ regional strike axis direction. The significance of the aforementioned statement depends on the stability of the rotation angle $\theta_{r}$ upon changing the measuring site, the period and the consideration of either the real or the imaginary parts. The rotation angle $\theta_{r}$ versus the period, calculated separately for the real and imaginary parts, for each of the sites $\mathrm{A}, \mathrm{B}$ and $\mathrm{C}$ is also depicted in Fig. 4. It is evident that the angle $\theta_{r}$ has (for all the measuring sites and for all the periods) approximately the same value $\theta_{r} \simeq 125^{\circ} \pm 5^{\circ}$, thus implying a stable direction striking $\sim \mathrm{N} 55^{\circ} \mathrm{W}$. This is further strengthened from the following facts: (i) this direction is close to one of the principal axes of the intrinsic coordinate system of the 2D regional geoelectric structure independently determined by the tensor decomposition analyses introduced by Bahr (Bahr, 1988) and Groom-Bailey, i.e., $\sim \mathrm{N} 40^{\circ} \mathrm{W}$; (ii) this direction is consistent to the strike direction determined 

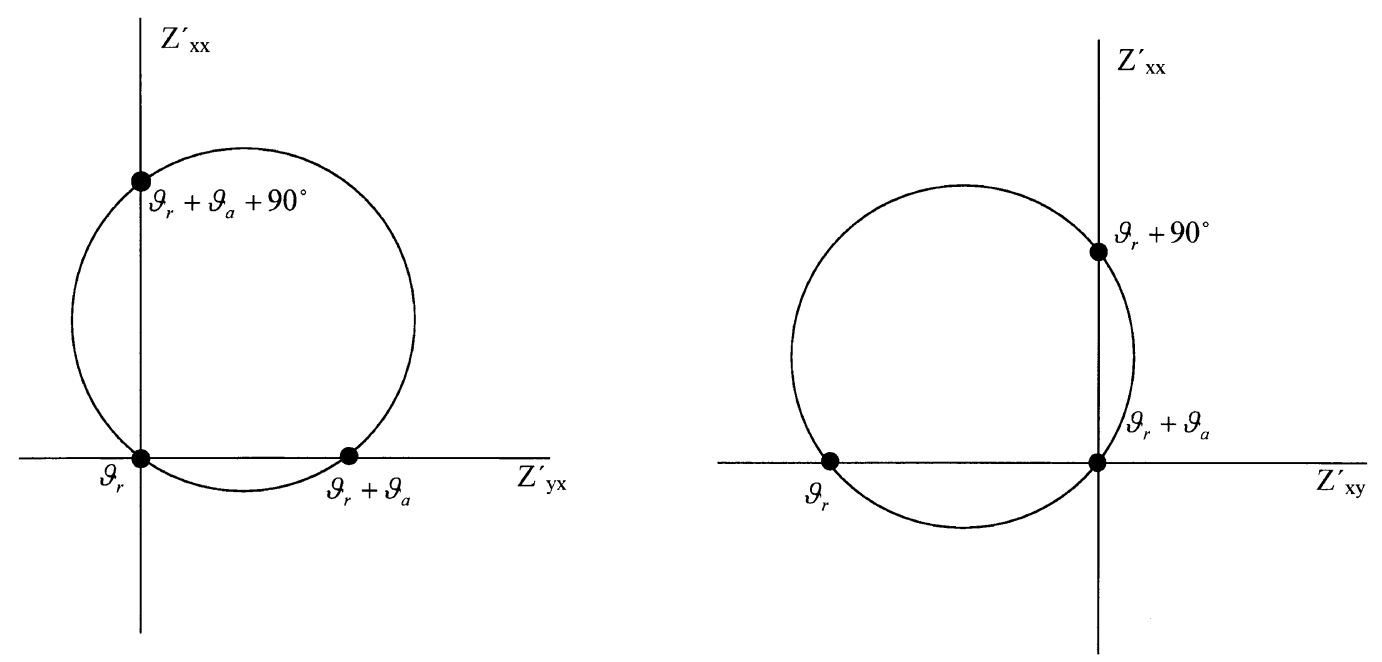

Fig. A.1. Solutions of angle $\theta$ derived from the model suggested in this paper for both the standard and "conjugate" forms of Mohr circle. Note that $\theta_{a}$ denotes the $\arctan \left(C_{1} / C_{3}\right)$.

by magnetic prospection when tipper and induction arrows analysis is used, i.e., $\sim \mathrm{N} 50^{\circ} \mathrm{W}$ (Makris et al., 1997). The fact that the direction of the polarisation of the electric field changes from site to site (see Figs. 4-5) provides further support that the aforementioned direction $\mathrm{N} 55^{\circ} \mathrm{W}$ exhibits a regional characteristic of the geoelectric structure. Furthermore, based on the physical model described above, i.e., a highly anisotropical $2 \mathrm{D}$ regional structure, we can lead to the determination of the regional stike angle (as it is shown in the Appendix). Finally, by adopting the physical model described above, we result to the following: the effect of linear polarisation seems to be a consequence of the specific conductivity conditions within the regional structure, regardless the existence or not of a current channelling in near surface distortion structures (cf. that the distortion tensor can not be uniquely determined, as it is shown in the Appendix).

\section{Conclusions}

The application of Mohr circles to MT data of Ioannina SES sensitive station determines the polarisation direction of the electric field at each measuring site; these results agree with those obtained from the raw data when the electric field polarisation diagrams are constructed. Furthermore, a new form of the magnetotelluric representation of Mohr circles is suggested, showing that the plot of $Z_{x x}^{\prime}(\theta)$ versus $Z_{y x}^{\prime}(\theta)$ determines in some cases the regional strike angle. This angle coincides with that determined by the decompositions of Bahr and Groom-Bailey; furthermore, it agrees with that angle determined by the tipper and induction arrows analysis when magnetic variation methods are pursued.

Acknowledgments. The authors are indebted to Dr. J. Pek for his detailed review of the paper which contributed a lot to its present form. Dr. F. E. M. Lilley is acknowledged for reading a manuscript of this paper and making useful comments and suggestions. This research was supported by the EC projects EV5V-CT94-0439 and EPET 388 (coordinated by Prof. P. Varotsos).

\section{Appendix}

Let us substitute $\mathbf{Z}_{\mathbf{m}}$ in Eq. (1) with the expression given in Eq. (A.1) of Groom and Bailey (1989). Since $Z_{B} \simeq 0$, according to the model suggested in Section 3, i.e., a highly anisotropical 2D regional structure, we obtain

$$
\begin{aligned}
\mathbf{Z}^{\prime}\left(\theta-\theta_{\mathbf{r}}\right)= & \mathbf{R}\left(\theta-\theta_{\mathbf{r}}\right) \underbrace{\left(\begin{array}{ll}
C_{1} & C_{2} \\
C_{3} & C_{4}
\end{array}\right)}_{\mathbf{C}\left(\theta_{\mathbf{r}}\right)} \\
& \times \underbrace{\left(\begin{array}{cc}
0 & Z_{E} \\
0 & 0
\end{array}\right)}_{\mathbf{Z}_{\mathbf{r}}\left(\theta_{\mathbf{r}}\right)} \mathbf{R}^{\mathbf{T}}\left(\theta-\theta_{\mathbf{r}}\right)
\end{aligned}
$$

with respect to the regional strike direction $\theta_{r}$. It is easy to show that for this model

$$
\begin{aligned}
& \text { If } Z_{x x}^{\prime}=0 \quad \text { then } \theta=\left\{\begin{array}{l}
\theta_{r} \\
\text { or } \\
\theta_{r}-\arctan \left(\frac{C_{1}}{C_{3}}\right)
\end{array},\right. \\
& \text { If } Z_{x y}^{\prime}=0 \quad \text { then } \theta=\left\{\begin{array}{l}
\theta_{r}+90^{\circ} \\
\text { or } \\
\theta_{r}-\arctan \left(\frac{C_{1}}{C_{3}}\right)
\end{array},\right. \\
& \text { If } Z_{y x}^{\prime}=0 \text { then } \theta=\left\{\begin{array}{l}
\theta_{r} \\
\text { or } \\
\theta_{r}-\arctan \left(\frac{C_{1}}{C_{3}}\right)+90^{\circ}
\end{array}\right.
\end{aligned}
$$

It is now clear that when we plot the "conjugate" form of Mohr circle, i.e., $Z_{x x}^{\prime}$ vs $Z_{y x}^{\prime}$, and the circle passes through the origin of the coordinate system, then this latter point corresponds to the regional strike angle, $\theta_{r}$. In this case the derivation of the regional strike angle from the standard Mohr circle, i.e., $Z_{x x}^{\prime}$ vs $Z_{x y}^{\prime}$, although not so evident, it is however possible, according to the solutions given above. Note also that these solutions involve only the elements of the first column of the distortion tensor, thus leading to incomplete information on this tensor. These solutions are illustrated in Fig. A.1. 


\section{References}

Bahr, K., Intepretation of the magnetotelluric impedance tensor: regional induction and local telluric distortion, Geophysics, 62, 119-127, 1988.

d'Erceville, I. and G. Kunetz, Some observations regarding naturally occuring electromagnetic fields in applied geophysics, Geophysics, 27, 651, 1962.

Fisher, G., L. Szarka, A. Adam, and J. T. Weaver, The magnetotelluric phase over 2-D structures, Geophys. J. Int., 108, 778-786, 1992.

Groom, R. W. and R. C. Bailey, Decomposition of magnetotelluric impedance tensors in the presence of local three-dimensional galvanic distortion, J. Geophys. Res., 94, 1913-1925, 1989.

Lilley, F. E. M. Diagrams for magnetotelluric data, Geophysics, 41, 766770,1976

Lilley, F. E. M. Magnetotelluric analysis using Mohr circles, Geophysics, 58, 1498-1506, 1993.

Makris, J., An electromagnetic study of the geoelectric structure of a region sensitive to the detection of pre-seismic electric signals, Ph.D. Thesis, University of Athens, 210 pp., 1997.

Makris, J., N. Bogris, and K. Eftaxias, Geoelectric structure of the VANstation at Ioannina sensitive to the detection of pre-seismic electric signals, in Practica of Athens Academy, Vol. 72, pp. 1-119, 1997.
Nye, J. F., Physical Properties of Crystals, 257 pp., Oxford Press, 1957.

Swift, M. C., Jr., Theoretical magnetotelluric and Turam response from two dimensional inhomogeneities, Geophysics, 36, 38-52, 1971.

Varotsos, P., M. Lazaridou, K. Eftaxias, G. Antonopoulos, J. Makris, and J. Kopanas, Short term earthquake prediction in Greece by pre-Seismic Electric Signals, in A critical Review of VAN, edited by Sir J. Lighthill, pp. 29-76, World Scientific, 1996a.

Varotsos, P., M. Lazaridou, K. Eftaxias, G. Antonopoulos, J. Makris, and J. Kopanas, Summary of the five principles suggested by Varotsos et al. (1996) and the additional questions raised in this debate, Geophys. Res. Lett., 23, 1449-1452, 1996 b.

Varotsos, P., N. Sarlis, M. Lazaridou, and P. Kapiris, Transmission of stress induced electric signals in dielectric media, J. Appl. Phys., 83, 60-70, 1998.

Varotsos, P., N. Bogris, K. Eftaxias, V. Hadjicontis, J. Makris, and M. Lazaridou, Seismic Electric Signals I. A detailed experimentation towards understanding the selectivity effect, Geophys. Res. Lett., 1999 (to appear).

J. Makris, N. Bogris (e-mail: nbogris@atlas.uoa.gr), and K.Eftaxias 